\title{
High Subsonic Flow Field from the Serpentine Nozzle
}

\author{
A Nageswara Rao', Rajat Arora ${ }^{2}$, Abhijit Kushari ${ }^{3}$ \\ ${ }^{1}$ Punjab Engineering College \\ Sector-12, Chandigarh, India \\ nagesh.aero63@gmail.com; rajatar@iitk.ac.in; \\ ${ }^{2}$ Indian Institute of Technology Kanpur \\ Kanpur, Uttar Pradesh, India \\ akushari@iitk.ac.in
}

\begin{abstract}
An experimental investigation is carried out to understand the flow-field and acoustic characteristics of the jets issuing from the serpentine nozzles at high subsonic Mach number 0.84. A microphone array was placed along the jet boundary to assess the acoustic characteristics. Near-field acoustic data shows that the OASPL pattern is asymmetric about the minor plane of the serpentine jet. OASPL pattern indicates the shear layer asymmetry about the minor plane of the serpentine jet. The proper orthogonal decomposition technique was performed on the schlieren data. The POD modes reveals the flapping motion of the jet about the minor plane of the serpentine jet.
\end{abstract}

Keywords: Schlieren, Microphone, Shear layer, OASPL, POD, Jet and acoustics.

\section{Introduction}

Deploying serpentine nozzle is gaining prominence as a passive device to shield the high-temperature components of the engine visually. The serpentine shapes have been extensively used at the exhaust of the jet engines in various fighter aircraft, such as the F-117A "Nighthawk" stealth bomber and "B-2 spirit" stealth strategic bomber. A considerable volume of research is available on the design parameters and the internal flow characteristics of the serpentine intakes and nozzles [1-2]. Liu et al. [3] have reported that a serpentine nozzle with a 2D rectangular exit could suppress IR signatures by $70 \%$ on an average when compared to a conventional round nozzle. However, there is no open literature available on the flow field and acoustic characteristics of the serpentine jets. The problem of flow through a serpentine nozzle with an elliptic exit is more complicated due to the complex duct geometry (flow transition from circular inlet to elliptic exit). Also, the flow field and acoustic characteristics of the serpentine nozzle increases the complexity of measurement of flow-field, using the conventional techniques. Therefore, in this paper we are proposing a naval technique to understand and analyse the spatial characteristics of the jet emanating from the serpentine nozzle. Also, the near-field acoustic studies are carried out to understand the acoustic propagation in the near-field of the jet. Therefore, the present study aims to improve the understanding of spatial flow-field as well as noise characteristics and rate of mixing of the jet exiting from serpentine nozzles, at transonic flow conditions.

\section{Experimental setup}

The measurements reported in the present investigation were carried out in an anechoic jet facility which was established at Aero propulsion laboratory, IIT Kanpur. Figure 1 show the photograph of the anechoic jet facility (refer Rajkumar et al. [4] and Rao and Kushari [5]). The acoustic jet facility comprise of a compressor, storage tank, venturi meter, a pressure regulating valve, and an anechoic chamber. The anechoic chamber was given access at two opposite ends to set up the open jet facility. The cut-off frequency was set to be $400 \mathrm{~Hz}$ based on the far-field characterization of the anechoic chamber. Figure 1, also shows a photograph of an anechoic chamber with the nozzle attached to a collar pipe. 


\subsection{Nozzle Details}

The serpentine nozzle used in the present investigation is a convergent nozzle with a circular inlet section of $25.4 \mathrm{~mm}$ inlet diameter, is mounted in the open jet facility using a collar pipe and flange arrangement. A smooth transition was given from the circular inlet to elliptic exit with the aspect ratio of 7.72. The orthographic views of the serpentine nozzle are shown in Fig. 2. The centerline of the nozzle is based on the Lee curve [6], which appears to be serpentine shape from the side view. The serpentine shapes can hide the high temperatures parts of the aircraft engine such as tail cone and turbine blades.

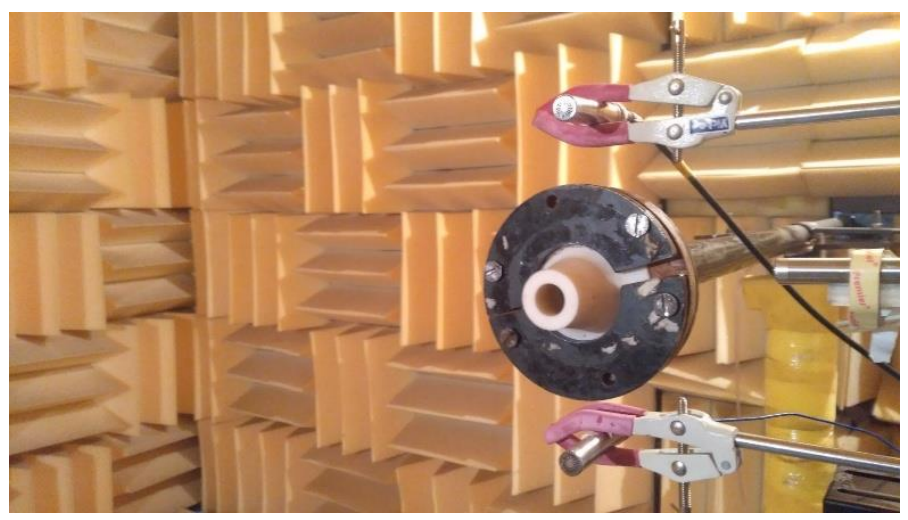

Fig. 1: Photograph of the anechoic chamber

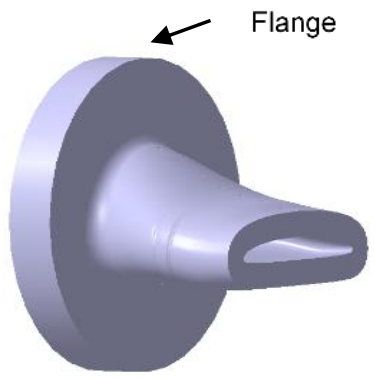

(a) Isometric view

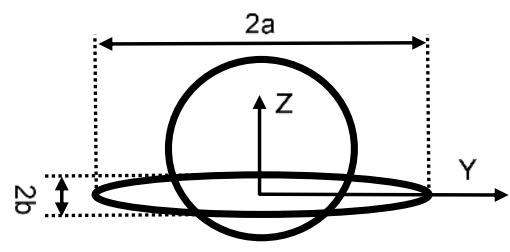

(c) Rear view

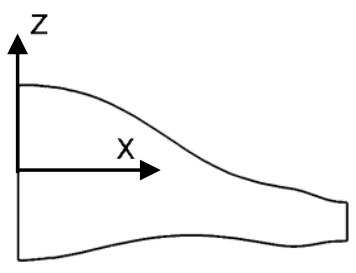

(b) Side view

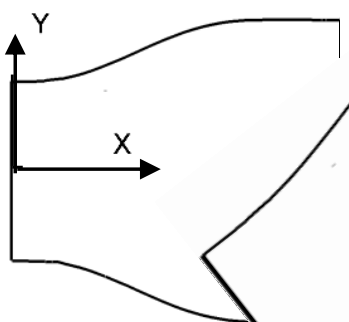

(d) Top view

Fig. 2: (a) Isometric view, (b) Side view, (c) Rear view, and (d) Top view of the serpentine nozzle 


\subsection{Measurement Techniques}

A microphone rake was designed and used to understand the noise characteristics along the near-field of the jet, as shown in Fig 3. A rake of four microphones, spaced 0.5 Deq apart were used for the near-field measurements. This microphone rake was mounted on a traversing system, and the measurements were taken at different locations over the rectangular grid. Thor labs three axes linear traversing system (Model no: LTS-300) was used to traverse the microphone rake. The near-field data acquired from the nozzle exit to 14Deq in the streamwise direction and 2 to 9 Deq in the radial direction. A PCB 130A24 1/2-inch microphone was used to measure the noise spectra. The microphone has a flat frequency response in the range of $20 \mathrm{~Hz}-16 \mathrm{kHz}$, within $\pm 1 \mathrm{~dB}$. The microphones were calibrated using a precision acoustic calibrator (LARSONDAVIS-CAL 200). The calibration was done at $1 \mathrm{kHz}$, for two output levels of $94 \mathrm{~dB}$ and $114 \mathrm{~dB}$. The sensitivity of the microphones found to be $10 \mathrm{mV} / \mathrm{Pa}$. The noise DAQ was interfaced with the LabVIEW software to monitor and record the data. The experiments were repeated three times to ensure the overall sound pressure level is within $\pm 1 \mathrm{~dB}$.

For visualizing the high subsonic jet, Z-type schlieren optical visualization technique was used. Schlieren uses refractive changes of light rays to perceive the deviations in the flow field. In the present study, the high-resolution images were recorded using the high-speed camera having a resolution of $400 \times 252$ pixels at a rate of 25000 frames per second (fps).

Snapshot POD is performed on time-resoled schlieren images. The refractive index gradient ( $\mathrm{n}$ ') is considered as the scalar field for the current analysis. Since, the POD is performed on the schlieren data set the energy content here is in terms of the mean square density gradient. The brief description about the proper orthogonal decomposition technique can be found in Nageswara Rao et al. [7].

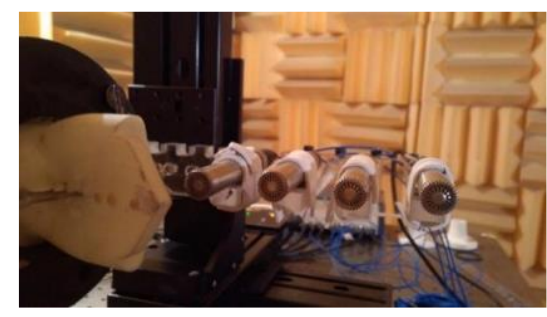

Fig. 3: Photograph of the microphone array

\section{Results and Discussion}

Figure 4 presents the near-field acoustic data for the serpentine nozzle. The jet emanating from a serpentine nozzle spreads asymmetrically across the minor plane (i.e. Z-axis), due to the nozzle curvature [5]. Therefore, to identify the effect of asymmetric jet development on the near-field acoustics, the measurements were carried out on either side (upper and lower) of the serpentine nozzle. A great care was taken, to ensure the distance is $\sim 2 \mathrm{D}_{\text {eq }}$ in the radial direction from the nozzle exit on either side during the measurement. There is a considerable difference observed in the OASPL pattern on the upper and lower sides of the jet for the serpentine nozzle, as shown in Fig. 4. The OASPL value on the lower side is $2 \mathrm{~dB}$ noisier than the upper side. From this OASPL pattern, it is evident and suggests that the formation of the streamwise vortices are relatively stronger on the lower side compared to the upper side. The peak noise region on the upper side is from $2 \mathrm{D}_{\text {eq }}$ to 8.5 $\mathrm{D}_{\text {eq }}$ while it is between $1.5 \mathrm{D}_{\text {eq }}$ to $9 \mathrm{D}_{\text {eq. }}$. The distinct variations in peak noise generation region on the upper and lower side of the jet deduces that the growth and thickness of the shear layer varying along the jet boundary. This emphasizes the strength of streamwise vortices is different, which further results in a difference in OASPL values on either side of the jet for the serpentine nozzle.

Figure 5 presents the first four high energetic POD modes based on their energy content. As it was mentioned earlier, the POD ranks the modes based on their energy and the energy content here is considered to be mean square energy density gradient. This is because the POD is applied to the schlieren data. First POD modes shows the jet oscillation in the radial direction, which may be attributable to the flapping motion. If we keenly observe the first POD mode, the jet oscillation is absent in the near-field of the jet (close to the nozzle exit) and appears to be present after a few downstream locations of the 
jet. This suggests that the jet entrainment may increase with the jet flapping, as the jet is propagating downstream. Also, the POD modes 2 to 4 suggests that the shear layer formation is not asymmetric about the minor plane of the jet. This may be due to the asymmetric shear layer thickness along the jet periphery. Figure 6 shows the time dependent coefficient correspond to the first four POD modes. PSD of first two POD modes reveal broad-band frequencies at $\sim 5 \mathrm{kHz}$. Therefore, the spatial structures correspond to the first two modes may be representing either the small scale structure formation in the flow field. PSD of mode 3 and 4 shows the propagation of structures in the downstream direction.
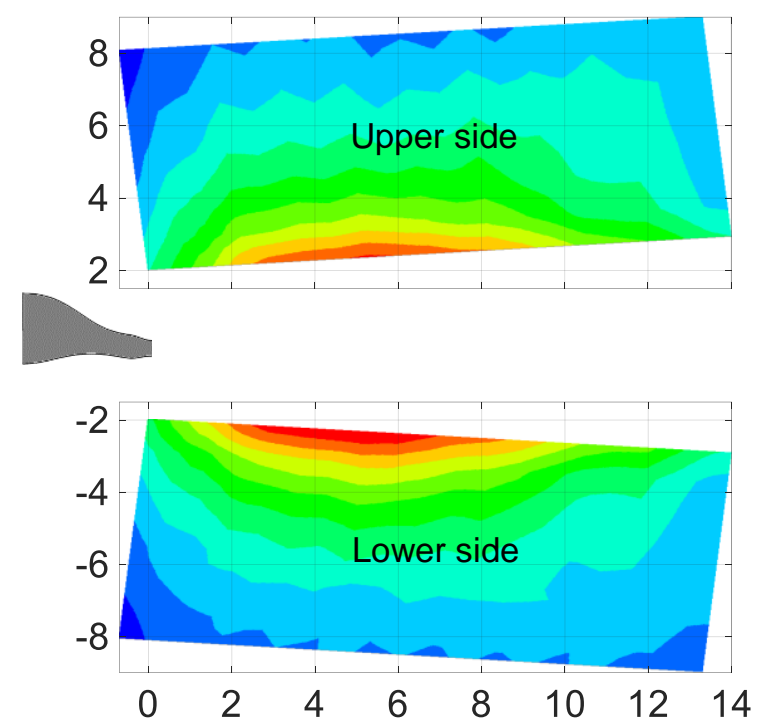

Fig. 4: OASPL contours in the near-field for serpentine nozzle at Mach number 0.84 

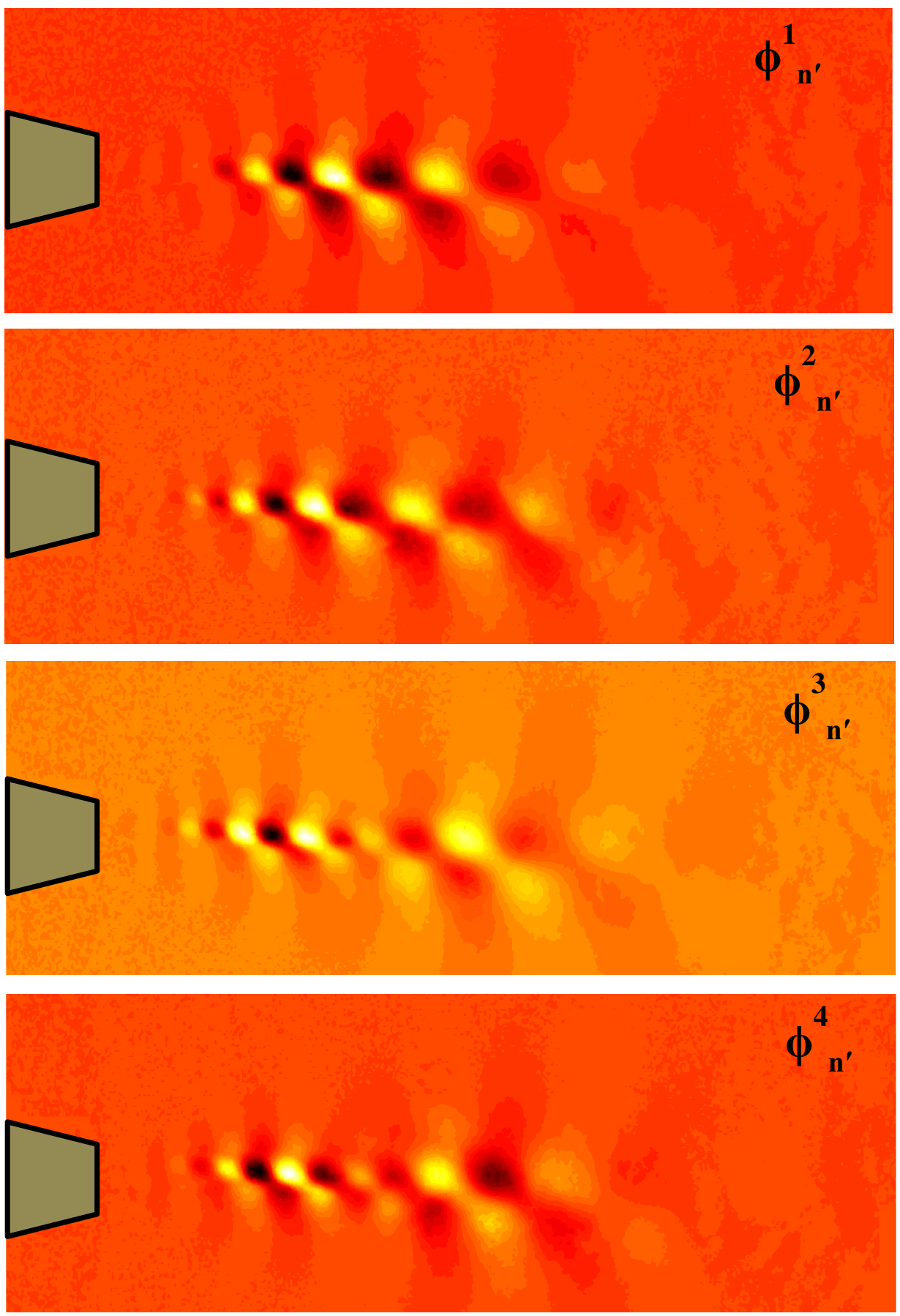

Fig. 5: First four high energetic modes in the minor plane of the serpentine nozzle 

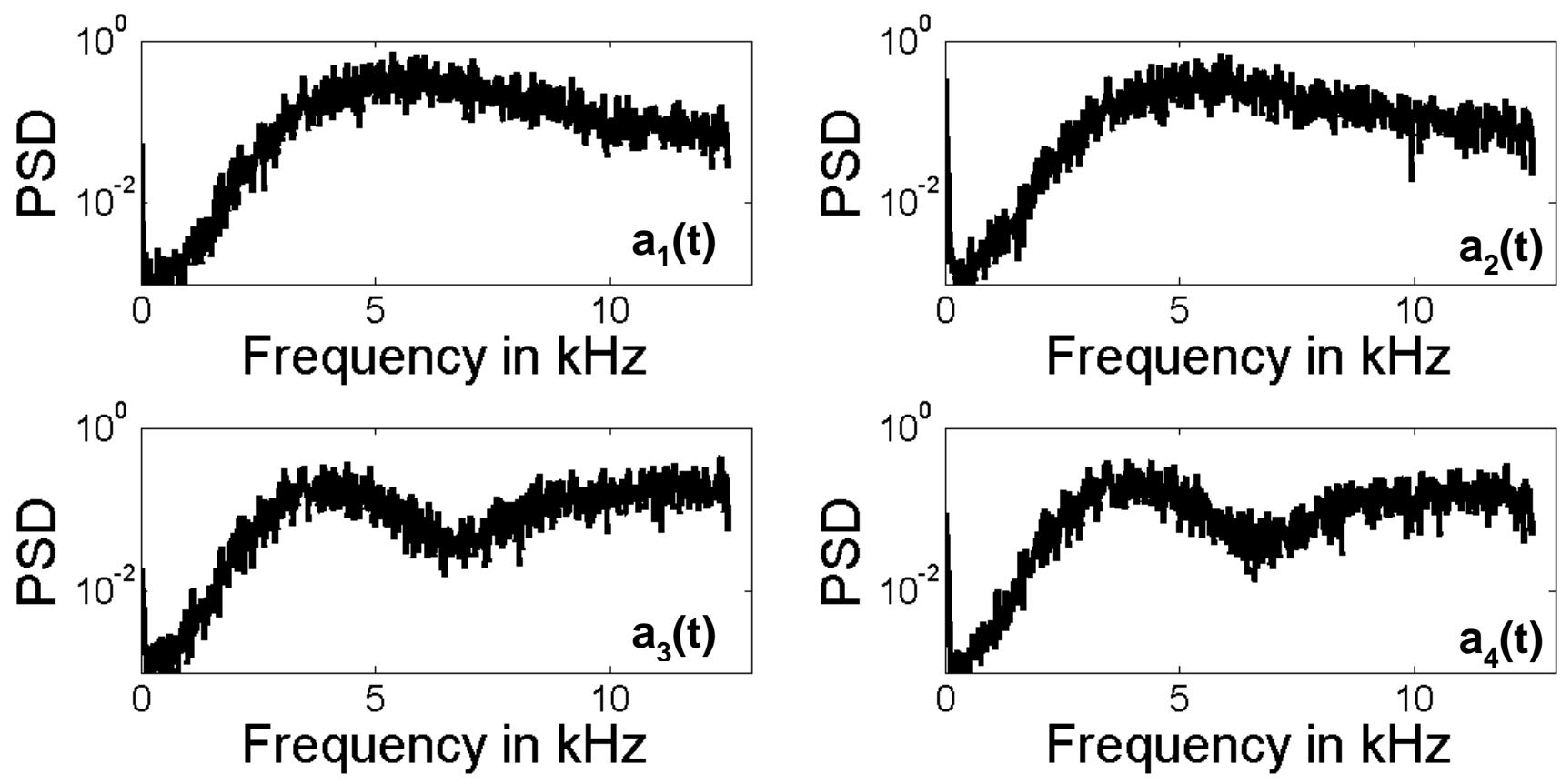

Fig. 6: Time dependent coefficients correspond to the POD modes

\section{Conclusion}

This study experimentally discussed the effect of the nozzle shape on the flow field and acoustic characteristics of high subsonic jet emanating from a serpentine nozzle at Mach number 0.84. Near-field OASPL mapping results indicate that the dominant noise is emanating at the end of the potential core break down. Also, the OASPL contours exhibit the asymmetric pattern in the minor plane of the serpentine nozzle. First few high energetic POD modes shows the oscillating nature of the jet in the minor plane, which is primarily identified as flapping. The near-field acoustic data, and POD modes, offer a good understanding of the flow-field from the complex serpentine nozzle.

\section{References}

[1] S. R. Wellborn, B.A. Reichert, T.H. Okiishi, "Study of the compressible flow in a diffusing s-duct," Journal of Propulsion and Power, 10(5) (1994) 668-675.

[2] X.L. Sun, Z.X. Wang, L. Zhou, J.W. Shi, Y.C. Zhang, "The design method of serpentine stealth nozzle based on coupled parameters," J. Eng. Thermophysics, 36(11) (2015) 2371-2375.

[3] C.C. Liu, H.H. Ji, W. Huang, F.F. Yang, "Numerical simulation on infrared radiation characteristics of serpentine 2-d nozzle," Journal of Aerospace Power. 28(7) (2013) 1482-1488.

[4] P. Rajkumar, T. Chandra Sekar, A. Kushari, B. Mody, B. Uthup, "Flow characterization for a shallow single serpentine nozzle with aft deck," Journal of Propulsion and Power. 33(5) (2017) 1130-1139.

[5] A.N. Rao, A. Kushari, G.K. Jaiswal, Effect of nozzle geometry on flow field for high subsonic jets, Journal of Propulsion and Power. 34(6) (2018) 1-13.

[6] C.C. Lee, C. Boedicker, "Subsonic diffuser design and performance for advanced fighter aircraft," in: Aircraft Design Systems and Operations Meeting, Colorado Springs, CO, AIAA-1985-3073, Oct., 1985.

[7] A. N. Rao, A. Kushari, and Alakesh Chandra Mandal, "Screech characteristics of under-expanded high aspect ratio elliptic jet," Physics of Fluids 32, no. 7 (2020): 076106. 